\title{
Multimedia on the web - editorial
}

\author{
Florian Stegmaier • Harald Kosch • Ralf Klamma • \\ Mathias Lux • Ernesto Damiani
}

Published online: 25 October 2013

(C) Springer Science+Business Media New York 2013

Streaming video has recently surpassed peer-to-peer networks in terms of network capacity hunger. Reports estimate a share of $40 \%$ of peak network capacity dedicated to entertainment, mostly streaming video. A large share of this traffic originates from web-based services. YouTube alone takes up to $8 \%$ of the prime time Internet traffic. While transmission currently works in a best effort system, multimedia information systems on the web are far from being perfect. Retrieval, annotation, validated and useful metadata, reliable and trusted services, and user interaction and context-based adaptation are still under discussion and issues to research.

Currently, the Web itself faces dramatic changes, looking for example at the spread of social networks, like Facebook, Twitter or the YouTube community, which become part of everyday life of more and more people. Technologies like Linked Data, HTML5, WebM, or WebRTC are enablers that make these changes possible and let people experience the Web without knowing details about technology. Also the availability of the Web has changed a lot. Many people create multimedia data on the go, put videos and photos on microblogs, or share audiovisual data with their loved ones or colleagues. These activities also have a deep effect on multimedia data formats and usage, multimedia systems and multimedia service providers. "Multimedia everywhere" was a common concept before, but this has changed to "relevant media at your finger tips", so new methods for finding, annotating, sharing, remixing, summarizing, transmitting, storing and consuming multimedia data have to be found.

F. Stegmaier $\cdot$ H. Kosch $(\bowtie)$

Lehrstuhl für Verteilte Informationssysteme, Universität Passau, Passau, Germany

e-mail: Harald.Kosch@uni-passau.de

F. Stegmaier

e-mail: Florian.Stegmaier@uni-passau.de

R. Klamma

Lehrstuhl für Informatik 5, RWTH Aachen University, Aachen, Germany

e-mail: klamma@dbis.rwth-aachen.de

M. Lux

Institute for Information Technology, Klagenfurt University, Klagenfurt, Austria

e-mail:mlux@itec.uni-klu.ac.at

E. Damiani

Department of Computer Science, Universita' degli Studi di Milano, Milan, Italy

e-mail: Ernesto.Damiani@unimi.it 
This special issue brings together research papers from the area of multimedia and the web discussing innovative ideas and new directions, new technologies, advances and challenges. The special issue call gained wide attention in the research community resulting in an overall amount of 24 papers submitted and the following 12 accepted manuscripts:

- "Experiencing Standardized Media Fragment Annotations within HTML5" by Davy Van Deursen, Wim Van Lancker, Erik Mannens and Rik Van de Walle. The combinations of two recent W3C recommendations of the "Video in the Web" activity as well as their extensions are in the focus of this manuscript. Following this proposal, rich media fragment annotations can be displayed in HTML5-based web environments.

- "Open Annotations on Multimedia Web Resources" by Bernhard Haslhofer, Robert Sanderson, Rainer Simon, and Herbert van de Sompel. The two W3C recommendations dealing with media fragmentation and annotation are not the only specification enabling the definition of semantically enriched media resources. In this manuscript, the current state of the Open Annotation Model is explained, design rationales are given, and it is aligned to create user annotations on multimedia Web resources.

- "CHM: an Annotation- and Component-based Hypervideo Model for the Web" by Madjid Sadallah, Olivier Aubert and Yannick Prié. Due to recent advances in standardization, hypervideos become more and more in fashion. This manuscript introduces an annotation-driven and component-based model to conceptualize hypervideos through a high level operational specification.

- "Intelligent Download and Cache Management for Interactive Non-Linear Video" by Britta Meixner and Jürgen Hoffmann. Beside the specification of hypervides and interactive media resources, one has to be aware of loading the media fragments in an efficient way. The manuscript is on a video framework that minimizes interruptions when the sequence of scenes is directly influenced by interaction, while the traditional viewing experience is not altered.

- "Semantic Enhancement for Media Asset Management Systems: Integrating the Red Bull Content Pool in the Web of Data" by Thomas Kurz, Georg Güntner, Violeta Damjanovic, Sebastian Schaffert, and Manuel Fernandez. Enrichment of media resources with open data stemming from the Linked Data cloud is a hot topic in the Semantic Web community. In this manuscript, the focus lies on automatic semantic enhancement services to link open accessible data media resources. Further, opportunities for media annotation, fragmentation and presentation are discussed in the context of the Red Bull Content Pool.

- "Building Mobile Multimedia Services: A Hybrid Cloud Computing Approach" by Dejan Kovachev, Yiwei Cao, and Ralf Klamma. Mobile multimedia services are in high demand, but their development comes at high costs. This manuscript introduces the I5CLoud, a hybrid cloud architecture, which serves as a substrate for scalable and fast time-to- market mobile multimedia services.

- "An Audio-Visual Approach to Web Video Categorization” by Bogdan Emanuel Ionescu, Klaus Seyerlehner, Ionut Mironica, Constantin Vertan, and Patrick Lam- 
bert. Due to the explosive spread of media resources on the Web, automatic approaches are envisioned to maintain this huge amount. This manuscript discusses and audiovisual approach to automatic web video categorization enlarged with relevance feedback.

- "With a Little Help from My Friends: Community-based Assisted Organization of Personal Photographs" by Claudio Cusano and Simone Santini. Another automatic approach to categorize still images is proposed in this manuscript. Here, a content-based method for semi-automatic organization of photo albums is introduced based on the analysis of how different users organize their own pictures. The goal is to help the user in dividing his pictures into groups characterized by a similar semantic content.

- "Non-Collaborative Content Detecting on Video Sharing Social Networks" by Antonio da Luz, Eduardo Valle, and Arnaldo de A. Araujo. Within the rise of social networks, control mechanisms related to media resources are more and more important. This manuscript is concerned with detecting non-collaborative content on video sharing social networks to avoid spamming activities.

- "Community and Trust-Aware Fake Media Detection" by Khaled Rashed, Dominik Renzel, Ralf Klamma, and Matthias Jarke. The Web itself is a very dynamic organism with uncountable interacting peers. The detection of fake media resources becomes more and more important and can be further improved by the consideration of trust.

- "Personalized vIdeo Adaptation Framework (PIAF): High-Level Semantics Adaptation” by Vanessa EL Khoury, David Coquil, Nadia Bennani, and Lionel Brunie. Video adaption is a cumbersome task, especially with the consideration of complex semantics based on user preferences. This paper places a significant emphasis on theoretical details of the utility function and the computation of the adaptation plan.

- "Sensory Effects for Ambient Experiences in the World Wide Web" by Markus Waltl, Christian Timmerer, Benjamin Rainer, and Hermann Hellwagner. Recent standardization work targeted towards a more comprehensive experience while watching media resources. To reach this goal, the Sensory Effect Metadata has been proposed to associate multimedia content with devices like fans, vibration chairs, or lamps.

Multimedia on the Web offers exciting challenges to researchers, deep technical problems to engineers, and fascinating application areas to Web designers. The wide range of challenges and opportunities was addressed in this special issue. Standardization and openness are keys to the still on going attractiveness of the Web. The possibility to exchange data, metadata and related services via standard Web browsers is facilitating a tremendous developer community and a large portion of the planet's population. Multimedia services like browser-based video conferencing are becoming are becoming a commodity in the moment and even advanced multimedia services like video editing can be offered on mobile smartphones utilizing cloud computing solutions. But the Web is still evolving and there are new opportunities on the horizon turning the web in a huge collaboration platform with more direct browser-to-browser communication in near real-time with standard mobile and social services. The trend that users have several Web-enabled devices will lead to new multi-device solutions seamlessly moving the user context on basis of standard Web protocols. The real power of multimedia on the Web is not unleashed yet. 


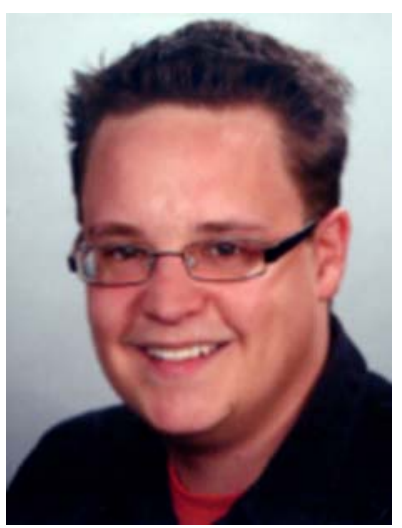

Florian Stegmaier Florian Stegmaier received his master degree at the University of Passau, Germany in 2008. As master thesis he implemented a Java based authoring tool for generating interactive videos on the basis of the Eclipse RCP serving as prototype of the iVi-Pro project. From November 2008 until April 2012, he was involved in the THESEUS Medico project as a third-party researcher. Since May 2012, he is leading the work package for Data Querying, Aggregation and Provenance in the FP7 CODE project. Further, he is also involved in MPEG/JPEG standardization and further an active member of the W3C Media Annotations Working Group as invited expert. Since November 2013, he is leading the work package for "Cross-Media Metadata Publishing" in the FP7 MICO project. In his $\mathrm{PhD}$, he is focusing on the following topics: heterogeneous and distributed multimedia retrieval, multimedia metadata and semantic web technologies.

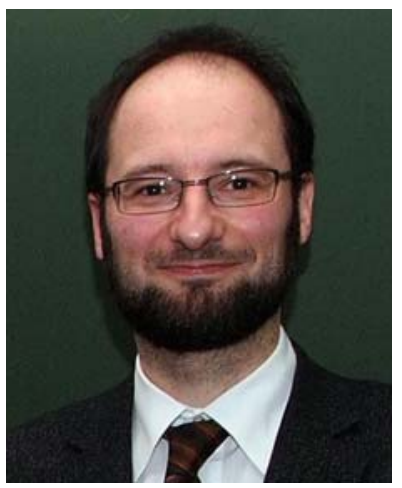

Harald Kosch Harald Kosch is a full professor and the head of the Chair of Distributed Information Systems at the University of Passau. His research topics are multimedia metadata, multimedia databases, middleware, and Internet applications. He started his research career at the Ecole Normale Supérieure and obtained his PhD in 1997. In 2002, he attained the habilitation at the University of Klagenfurt. In 2006, he took over the Chair of Distributed Information Systems at the University of Passau. Harald Kosch successfully participated in the MPEG-7, W3C and the MPEG-21 standardization process, is one of the co-founders of the multimedia metadata community, and owns several patents. He has been organizing many international conferences and workshops covering different aspects of multimedia engineering. 


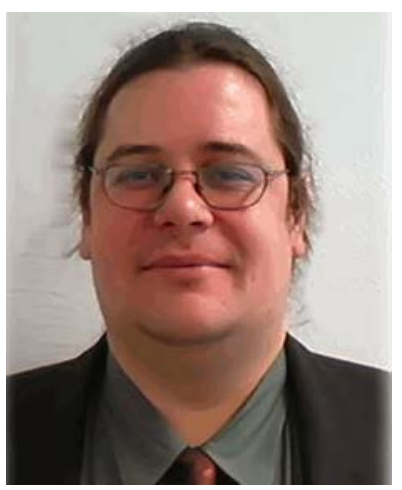

Ralf Klamma Ralf Klamma has diploma, doctoral and habilitation degrees in computer science from RWTH Aachen University. He leads the research group "advanced community information systems" (ACIS) at the information systems chair, RWTH Aachen University. He is coordinating and working in major EU projects for Technology Enhanced Learning (Learning Layers, GALA, METIS and BOOST). He is member of the research excellence cluster "Ultra High Speed Mobile Information and Communication" (UMIC) specialized in mobile multimedia. Ralf organized doctoral summer schools and conferences in Technology Enhanced Learning, and Social Network Analysis. He is on the editorial board of IEEE Transactions on Technology Enhanced Learning and Social Network Analysis and Mining (SNAM). His research interests are community information systems, multimedia metadata, social network analysis and technology enhanced learning.

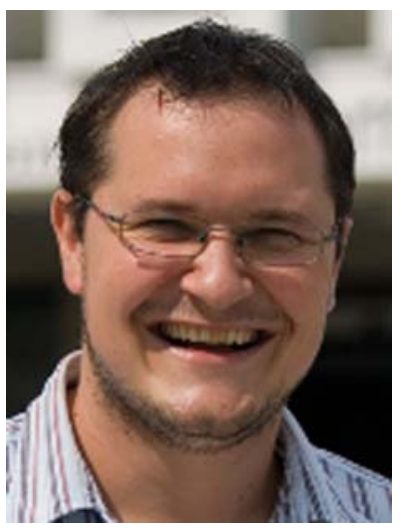

Mathias Lux Dr. Mathias Lux is Senior Assistant Professor at the Institute for Information Technology (ITEC) at Klagenfurt University. He is working on user intentions in multimedia retrieval and production and emergent semantics in social multimedia computing. Mathias Lux received his M.S. in Mathematics 2004, his Ph.D. in Telematics 2006 from Graz University of Technology, both with distinction, and his Habilitation (venia docendi) from Klagenfurt University in 2013. In his scientific career he has (co-) authored more than 80 scientific publications, has served in multiple program committees and as reviewer of international conferences, journals and magazines, and has organized multiple scientific events. Mathias Lux is also well known for the development of the award winning and popular open source tools Caliph \& Emir and LIRE (http:// www.semanticmetadata.net) for multimedia information retrieval. 


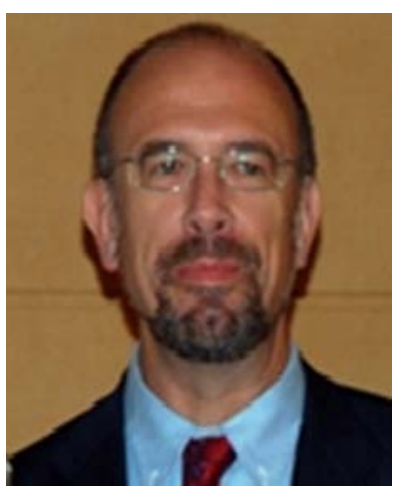

Ernesto Damiani Prof. Ernesto Damiani is a full professor at the Università degli Studi di Milano and the director of the Università degli Studi di Milano's PhD program in computer science. He has also done extensive research on advanced network infrastructure and protocols, taking part in the design and deployment of secure high-performance networking environments, both as chief scientist and in management positions. His areas of interest include Cloud and Web services security, processing of semi and unstructured information (e.g., XML), and semantics-aware content engineering for multimedia. He has published several books and about 200 papers and international patents. His work has appeared, among others, in the IEEE Transactions on Knowledge and Data Engineering, the ACM Transactions on Information and System Security, and TFS, as well as in the ACM Transactions on Information Systems and the ACM Transactions on Software Engineering and Methodology. According to Google Scholar, Ernesto Damiani's publications have more than 6800 citations. Prof. Damiani has served and is serving in all capacities on many congress, conference, and workshop committees. He is a senior member of the IEEE and ACM distinguished scientist. Ernesto Damiani leads/has led a number of international research projects: he is the Principal Investigator of the ASSERT4SOA project (STREP) on the security certification of service oriented architectures; leads the activity of SESAR research unit within SecureSCM (STREP), ARISTOTELE (IP), and CUMULUS (STREP) projects funded by the European Commission in the Seventh Framework Program. He has been an Associate Editor of the IEEE Transactions on Service-Oriented Computing since its inception. He has served as Area Editor of the Journal of System Architecture (Elsevier). Also, Prof. Damiani is Editor in-chief of the International Journal of Knowledge and Learning (Inderscience) and of the International Journal of Web Technology and Engineering (IJWTE). 